\title{
Zinc-containing Vaginal Moisturizer Gel Improves Postmenopausal Vulvovaginal Symptoms: A Pilot Study
}

\author{
Peter Takacs ${ }^{1}$, Bence Kozma², Balazs Erdodi', Attila Jakab², Kindra Larson', Robert Poka ${ }^{2}$ \\ ${ }^{1}$ Division of Female Pelvic Medicine and Reconstructive Surgery, Department of Obstetrics and Gynecology, Eastern Virginia Medical School, \\ Norfolk, VA, USA, ${ }^{2}$ Department of Obstetrics and Gynecology, University of Debrecen Faculty of Medicine, Debrecen, Hungary
}

Objectives: Although vaginal estrogen is highly effective in alleviating genitourinary symptoms of menopause (GSM), some women are reluctant to use hormonal treatment. Our aim was to evaluate the effect of a zinc-containing vaginal moisturizer gel on GSM.

Methods: Women with GSM were enrolled. Patients were asked to use the vaginal gel daily for 2 weeks. Vaginal Health Index (VHI) was calculated. Visual analogue scale (VAS) was obtained on vaginal pain, dryness, burning, itching, dyspareunia and dysuria before and 2 weeks after the completion of treatment. Vaginal cytology was obtained. Vulvovaginal symptom questionnaire (VSQ21) was used to assess subjective symptoms.

Results: Thirty-seven women with GSM participated in the study. The VHI improved significantly (mean \pm standard deviation, $13 \pm$ 4 before vs. $17 \pm 4$ after treatment, $P<0.01$ ). The patient reported VAS combined score was significantly lower after the treatment (18 \pm 14 before vs. $7 \pm 12$ after treatment, $P<0.01$ ). The largest improvement occurred in vaginal dryness. The maturation value did not change significantly. There were no signs of inflammation on vaginal cytology. VSQ-21 combined scores also improved significantly $(7.9 \pm 5.5$ before vs. $2.7 \pm 3.6$ after the intervention, $P<0.01)$. Side effects were rare and minor.

Conclusions: Novel zinc-containing vaginal moisturizer gel significantly improves postmenopausal vulvovaginal symptoms without serious side effects. (J Menopausal Med 2019;25:63-68)

Key Words: Zinc · Vagina · Menopause

\section{Introduction}

Vulvovaginal atrophy (VVA) is a prevalent condition affecting many postmenopausal women. ${ }^{1}$ Up to $40 \%$ of postmenopausal women develop symptoms of $\mathrm{VVA}^{2.5}$ It is most often diagnosed by its symptoms, including vaginal dryness, burning, itching, vagina pain and dyspareunia. More recently these symptoms have been described as a syndrome: the genitourinary syndrome of menopause (GSM). ${ }^{6,7}$ This new term describes various menopausal symptoms and signs including not only genital symptoms but sexual (pain) and urinary symptoms (dysuria, urgency) as well. The pathophysiology of GSM/VVA can be explained by the decline in estrogen levels associated with diminished ovarian function and natural aging.

Traditionally the treatment options for GSM/VVA was vaginal estrogen supplementation but many women are either unable to or are afraid to use hormones. There are several nonhormonal treatment modalities for the management of VVA related symptoms, especially for one of the most

Received: December 5, 2018 Revised: Febuary 19, 2019 Accepted: March 4, 2019

Address for Correspondence: Peter Takacs, Division of Female Pelvic Medicine and Reconstructive Surgery, Department of Obstetrics and Gynecology, Eastern Virginia Medical School, 825 Fairfax Avenue, Suite 526 Norfolk, VA 23507-2007, USA

Tel: +1-757-446-7131, Fax: +1-757-446-7088, E-mail: takacsp@evms.edu

ORCID: https://orcid.org/0000-0002-0078-5763 
bothersome symptoms of vaginal dryness. The 2013 position statement of The North American Menopause Society indicates that the first-line therapies to alleviate symptoms of VVA should include nonhormonal vaginal lubricants and moisturizers as well as regular sexual activity. ${ }^{8}$ Although there are numerous vaginal moisturizers and lubricants are commercially available only a few of those have been tested in clinical trials.

Previously we investigated the role of vaginal zinc supplementation on vaginal remodeling. ${ }^{9}$ We showed that in human vaginal smooth muscle cells, zinc has a beneficial effect on the production of extracellular components produced by the muscle at $20 \mu \mathrm{M}$ zinc tissue level, thereby increasing the amount of elastin production. ${ }^{10}$ Earlier animal studies have shown that zinc plays an important role in the vaginal extracellular matrix (ECM) composition. When rats were kept on a zinc-lacking diet, the vaginal structure became similar to the menopausal vagina. ${ }^{11}$ With vaginal zinc replacement, the vaginal ECM could be regenerated with characteristics equivalent to juvenile rats. ${ }^{9}$

The primary aim of this pilot study was to test the effect of a novel zinc-containing vaginal gel on GSM/ VVA symptoms. Secondary objectives included identifying potential side effects and safety concerns with its use.

\section{Materials and Methods}

A prospective cohort study was performed with the enrollment of women with GSM at the outpatient clinic of the Department of Obstetrics and Gynecology, University of Debrecen, Hungary between March 2017 and March 2018. Our study was approved by the Hungarian National Institutional Review Medical Research Council. Patients were enrolled after signed, informed consent was obtained (approval no. 7239-3/2017EIUG). Enrollment criteria included the presence of symptoms of vulvovaginal atrophy/GSM in postmenopausal women. To be defined as a postmenopausal woman, individuals had to have at least 12 consecutive months of amenorrhea without any other obvious reason/ or consistently elevated follicle-stimulating hormone blood levels of $30 \mathrm{mIU} / \mathrm{mL}$ or higher. Exclusion criteria were premenopausal state, local or systemic hormone therapy within the past six months, vaginal infection at presentation, cytological atypia, prior radiation treatment, history of breast, ovarian or other gynecological cancer, pelvic organ prolapse $>$ stage 2 , and the recent use (3 months) of any vaginal product or douching.

Patients were asked to use commercially available zinc containing vaginal gel (JUVIA vaginal gel; Fempharma Europe, LLC., Budapest, Hungary) daily for 2 weeks. The key ingredients of the gel are water, hydroxyethyl cellulose, zinc sulfate, and lactic acid. The novel gel was selfapplied intra vaginally via a vaginal applicator. Participants were asked to place $2 \mathrm{~mL}$ of gel into the vagina nightly for 2 consecutive weeks. Patients underwent a detailed gynecological exam at enrollment and 2 weeks after completing the treatment (about 4 weeks after enrollment). At the first visit general gynecological and medical history was taken (age, body mass index, previous deliveries or operations, menstruation cycle, the onset of menopause, and hormonal therapy). Demographic and pertinent clinical information was recorded prospectively and stored in a dedicated database.

Postmenopausal women with symptoms of GSM were asked to complete a questionnaire reporting their symptoms (vaginal pain, dyspareunia, vaginal dryness, burning, itching, and dysuria), and mark the severity of their symptoms on a 0-10 visual analogue scale (VAS). A score of 0 indicated the absence of a symptom and a score of 10 the worst possible symptoms. Also, a validated vulvovaginal symptom questionnaire (VSQ-21) was used to assess subjective symptoms before and 2 weeks after treatment. ${ }^{12}$ The clinical evaluation was performed by a board-certified obstetrician and gynecologist blinded to the specific study related information. The clinical data gathered included components of the Vaginal Health Index (VHI) score: elasticity, fluid secretion, $\mathrm{pH}$ and epithelial mucosa (integrity) and moisture components. Each component is scored on a scale of 1 (worst) to 5 (best). ${ }^{13}$ Lower scores indicate more severe atrophy. VHI was calculated at baseline and 2 weeks after the completion of treatment. In addition, a vaginal smear was obtained from the right lateral vaginal wall using a spatula and stained according to the Papanicolaou technique at baseline and 2 weeks after completion of the intervention. The cytology samples 
were evaluated by an independent, blinded, board-certified pathologist trained and authorized to review vaginal cytology. The Vaginal Maturation Index (VMI) was evaluated by calculating the percentage of superficial, intermediate and parabasal epithelial cells on the smear. Subsequently, we calculated the vaginal maturation values $($ VMV) $[(1 \times$ \%superficial $)+(0.5 \times$ \%intermediate $)+(0 \times \%$ parabasal $)]^{1}$ Study participants completed an online, anonymous, selfadministered questionnaire to give an opportunity to provide feedback after completion of the study. Those questions included: Q1-Have you experienced any side effects during or after completion of the treatment? (Answers: Yes or No). Q2-How satisfied were you with the moisturizing effect of the gel? (Answers: Likert-type scale 1-5). Q3-For how long did the moisturizing effect last after stopping the treatment? (Answer: days). Q4-How comfortable did you feel using the gel? (Answers: Likert-type scale 1-5). Q5-Did your vulvovaginal symptoms improve significantly? (Answers: Yes or No). Q6-Did you feel more self-confident after using the gel? (Answers: Yes or No). Q7-How satisfied were you with the gel? (Answers: Likert-type scale 1-5). Q8-Were you satisfied with the consistency of the gel? (Answers: Yes or No). Q9Were you satisfied with the smell of the gel? (Answers: Yes or No).

Statistical analysis was performed with SigmaStat/SPSS software (Systat Software Inc., San Jose, CA, USA). To de-

Table 1. Clinical and demographic characteristics of the study sample

\begin{tabular}{lc}
\hline \multicolumn{1}{c}{ Variable } & Data \\
\hline Subjects & $37(100)$ \\
Age $(\mathrm{y})$ & $60 \pm 9$ \\
Gravida & $3 \pm 2$ \\
Para & $2 \pm 1$ \\
Body mass index $\left(\mathrm{kg} / \mathrm{m}^{2}\right)$ & $27 \pm 4$ \\
Duration in menopause $(\mathrm{y})$ & $11 \pm 9$ \\
Prior C-section & $5(13.5)$ \\
Prior hysterectomy/BSO & $8(21.6)$ \\
\hline
\end{tabular}

The data is presented as number (\%) or mean \pm standard deviation.

Gravida: number of pregnancies, Para: number of deliveries, Csection: caesarean section, BSO: bilateral salpingo-oophorectomy. scribe the clinical and demographic characteristics, means \pm standard deviation (SD) were used for continuous variables. Paired $t$ test was used to compare differences before and after treatment results. Differences were considered significant when $P$ value was less than 0.05 . Data are presented as mean values $( \pm \mathrm{SD})$ if not otherwise specified.

\section{Results}

All patients were in menopause. The average age was 60 \pm 9 years, and on average $11 \pm 9$ years had passed since their last menstrual period. The rest of the demographic information is as described in Table 1. The VHI improved significantly (13 \pm 4 before vs. $17 \pm 4$ after completion of treatment, $P<0.01$; Table 2). The largest incremental VHI component change occurred in vaginal moisture. In sixty percent of the women, the vaginal $\mathrm{pH}$ remained normalized $(\mathrm{pH}<4.6)$ even 2 weeks after cessation of treatment.

The patient reported VAS combined score was significantly lower after the treatment $(18 \pm 14$ before vs. $7 \pm 12$ after treatment, $P<0.01$; Table 3 ). The largest improvement occurred in vaginal dryness, followed by dyspareunia, burning and itching.

The VMI and VMV did not change significantly (Table 4). There were no signs of inflammation on vaginal cytology.

VSQ-21 combined scores also improved significantly (7.9 \pm 5.5 before vs. $2.7 \pm 3.6$ after the intervention, $P<0.01$ ). Again, scores significantly lower indicate symptom improvement.

Table 2. Vaginal Health Index before and after treatment with the vaginal gel

\begin{tabular}{lccc}
\hline \multicolumn{1}{c}{ Variable } & $\begin{array}{c}\text { Before treatment } \\
(\mathrm{n}=37)\end{array}$ & $\begin{array}{c}\text { After treatment } \\
(\mathrm{n}=37)\end{array}$ & $P$ value \\
\hline Elasticity & $2.8 \pm 0.9$ & $3.6 \pm 1.0$ & $<0.01$ \\
Fluid secretion & $2.5 \pm 0.8$ & $3.4 \pm 1.0$ & $<0.01$ \\
Vaginal pH & $2.6 \pm 1.7$ & $2.9 \pm 1.7$ & 0.47 \\
Epithelial mucosa & $2.8 \pm 0.9$ & $3.7 \pm 1.0$ & $<0.01$ \\
Moisture & $2.7 \pm 1.0$ & $3.5 \pm 1.1$ & $<0.01$ \\
Total score & $13.2 \pm 4.3$ & $17.1 \pm 5.1$ & $<0.01$ \\
\hline
\end{tabular}

The data is presented as mean \pm standard deviation. 
Table 3. VAS score before and after treatment with the vaginal gel

\begin{tabular}{lccc}
\hline \multicolumn{1}{c}{ Variable } & $\begin{array}{c}\text { Before treatment } \\
(\mathrm{n}=37)\end{array}$ & $\begin{array}{c}\text { After treatment } \\
(\mathrm{n}=37)\end{array}$ & P value \\
\hline Vaginal pain & $2.1 \pm 3.3$ & $1.3 \pm 2.7$ & 0.29 \\
Burning sensation & $3.1 \pm 3.6$ & $0.9 \pm 2.3$ & $<0.01$ \\
Itching & $2.4 \pm 5.3$ & $0.8 \pm 2.4$ & 0.02 \\
Vaginal dryness & $5.4 \pm 3.7$ & $2.0 \pm 2.8$ & $<0.01$ \\
Dyspareunia & $5.0 \pm 4.2$ & $2.0 \pm 3.0$ & $<0.01$ \\
Dysuria & $0.7 \pm 1.7$ & $0.31 \pm 1.1$ & 0.32 \\
Total score & $18.2 \pm 14.8$ & $7.0 \pm 12.4$ & $<0.01$ \\
\hline
\end{tabular}

The data is presented as mean \pm standard deviation.

VAS: visual analogue scale (0-10, lower scores indicates less bother).

Based on the anonymous online questionnaire, 97\% reported that during treatment their vagina was well moisturized and 94\% felt that the treatment was very comfortable. The moisturizing effect lasted for at least for 1 week after cessation of treatment in $75 \%$ of women. Eighty-two percent of participants felt more self-confident after using the gel and reported a significant improvement in the quality of the sexual relationship. Overall $92 \%$ of women were extremely satisfied or satisfied with the vaginal gel treatment. The consistency of the gel was satisfactory (not too thick or too liquid) to $85 \%$ of woman and every woman agreed that the smell of the gel was satisfactory. Side effects were rare and minor. Two women reported increased vaginal discharge. One of those women discontinued the treatment and her symptoms resolved after that.

\section{Discussion}

Our prospective cohort pilot trial revealed that a novel zinc-containing vaginal moisturizer gel significantly improves postmenopausal vulvovaginal symptoms without serious side effects. The VHI, the patient reported VAS and VSQ-21 scores improved significantly. The largest VAS improvement occurred in vaginal dryness, and not surprisingly the largest incremental change occurred in vaginal moisture out of the VHI components. We did not see a change in the VMI
Table 4. Vaginal cytology with Maturation Index and maturation values before and after treatment with the vaginal gel

\begin{tabular}{lccl}
\hline \multicolumn{1}{c}{ Variable } & $\begin{array}{c}\text { Before treatment } \\
(\mathrm{n}=37)\end{array}$ & $\begin{array}{c}\text { After treatment } \\
(\mathrm{n}=37)\end{array}$ & $P$ value \\
\hline Maturation Index & & & \\
Parabasal (\%) & $26 \pm 37$ & $24 \pm 33$ & 0.86 \\
Intermediate (\%) & $64 \pm 32$ & $62 \pm 30$ & 0.78 \\
Superficial (\%) & $10 \pm 13$ & $14 \pm 19$ & 0.39 \\
Maturation value & $42 \pm 22$ & $45 \pm 22$ & 0.66 \\
\hline
\end{tabular}

The data was presented as mean \pm standard deviation. None of the specimens showed signs of inflammation.

or VMV consistent with the nonhormonal nature of the gel. There were no signs of inflammation on vaginal cytology and side effects were rare and minor.

Nonhormonal treatment options for VVA symptoms include lubricants (water, silicone or oil based) and moisturizers. Recently other treatment options for the alleviation of atrophy related symptoms were tried. ${ }^{14}$ Regular use of nonhormonal, long-acting vaginal moisturizing agents can significantly improve symptoms of vaginal dryness, decrease vaginal $\mathrm{pH}$ to premenopausal levels without affecting VMI but not as effectively as hormonal treatments. ${ }^{15-17}$ Although several lubricants and moisturizers are commercially available over the counter, very few clinical trials have been performed to show their safety and efficacy. ${ }^{15-18}$ In one study that did examine the safety of personal moisturizers and lubricants, investigators found that many water-based gels are hyperosmolar and may lead to epithelial cell damage and toxicity. ${ }^{18}$ The vaginal gel tested in our pilot trial has been rigorously evaluated and found to be nontoxic to epithelial cells and not hyperosmolar (unpublished data based on communication with the manufacturer). Our clinical evaluation agrees with that. We have found no signs of inflammation on pelvic exam or evidence of increased inflammatory cells on cytology. In fact, we did not see any change in the cytology at all (VMI, VMV, or evidence of inflammation). Two patients reported increased vaginal discharge, but none had evidence of infection or vaginal irritation on pelvic exam. No other gel related side effects were reported.

There are several potential mechanisms how the tested gel may have caused the improvement of the GSM-related 
symptoms. All of the key components of the gel could have contributed to the symptom improvement via different mechanisms. The formulation is based on water and hydroxyethyl cellulose polymer which can bind to the vaginal mucosa and keep the moisture on the epithelial surface. The lactic acid provides the appropriate acidic $\mathrm{pH}$ for maintaining the necessary vaginal environment favored by lactobacilli. The zinc sulfate heptahydrate complex binds 7 water molecules providing the necessary hydration to the atrophic vagina and zinc can also penetrate the vaginal mucosa and may reach the lamina propria and muscularis layer of the vagina. $^{19}$

We have previously shown the beneficial effects of zinc on human cultured vaginal smooth muscles cells. Under culture conditions, we were able to significantly stimulate the vaginal smooth muscle cell elastin production within 48 hours after exposure to $20 \mu \mathrm{M}$ of zinc sulfate. ${ }^{10}$ In addition, in a rat model, we were able to improve upon the quality of the vaginal wall by vaginal zinc supplementation for 2 weeks in oophorectomized rats. ${ }^{9}$ The vaginal wall (epithelium, lamina propria, and muscularis) was significantly thicker, the ratio of vaginal collagen I/III and I/V expressions was significantly up-regulated compared to placebo after the application of zinccontaining vaginal suppositories. ${ }^{9}$

There are several limitations to our pilot study. First and most importantly we are limited by the small number of women enrolled. In addition, the short-term nature of the study precludes our ability to assess the long-term effects of the gel. The design of the study (single cohort without a comparison group) does not allow us to conclude whether a specific ingredient or if the placebo effect is responsible for the improved clinical signs and patient-reported subjective symptoms. Only a large-scale, randomized, placebo-controlled trial could definitively answer those questions. Lastly, even though the online, anonymous self-reported questionnaire gave an opportunity for our study participants to report on their experiences with the vaginal gel, this questionnaire has not been validated.

In summary, the novel zinc-containing vaginal moisturizing gel has significantly improved the GSM/VVA symptoms of postmenopausal women. With this pilot study, we have created the foundation for a larger comparative study. We plan to conduct a larger scale, randomized, placebo-controlled trial in the near future to assess the benefits of a zinc-containing vaginal gel compared with both a placebo group and an estrogen-containing vaginal gel group.

\section{Acknowledgements}

This research was supported by Economic Development and Innovation Operational Program Grant of the European Union and Hungary (GINOP-2.1.1-15-2016-00783).

\section{Conflict of Interest}

Takacs is a paid consultant for Fempharma LLC. No other authors have no potential conflict of interest relevant to this article was reported.

\section{References}

1. Weber MA, Limpens J, Roovers JP. Assessment of vaginal atrophy: a review. Int Urogynecol J 2015; 26: 15-28.

2. Bachmann GA, Nevadunsky NS. Diagnosis and treatment of atrophic vaginitis. Am Fam Physician 2000; 61: 3090-6.

3. Levine KB, Williams RE, Hartmann KE. Vulvovaginal atrophy is strongly associated with female sexual dysfunction among sexually active postmenopausal women. Menopause 2008; 15: 661-6.

4. Nappi RE, Kokot-Kierepa M. Women's voices in the menopause: results from an international survey on vaginal atrophy. Maturitas 2010; 67: 233-8.

5. Shobeiri F, Jenabi E, Hazavehei SM, Roshanaei G. Quality of life in postmenopausal women in Iran: a populationbased study. J Menopausal Med 2016; 22: 31-8.

6. Gandhi J, Chen A, Dagur G, Suh Y, Smith N, Cali B, et al. Genitourinary syndrome of menopause: an overview of clinical manifestations, pathophysiology, etiology, evaluation, and management. Am J Obstet Gynecol 2016; 215: 704-11.

7. Kim HK, Kang SY, Chung YJ, Kim JH, Kim MR. The recent review of the genitourinary syndrome of menopause. $\mathrm{J}$ Menopausal Med 2015; 21: 65-71.

8. Management of symptomatic vulvovaginal atrophy: 2013 position statement of The North American Menopause So- 
ciety. Menopause 2013; 20: 888-902; quiz 9034.

9. Takacs P, Jaramillo S, Zhang Y, Datar R, Williams A, Olczyk $J$, et al. The effects of PPAR $\delta$ agonist and zinc on ovariectomized rats' vagina. Female Pelvic Med Reconstr Surg 2013; 19: 126-31.

10. Takacs P, Zhang Y, Candiotti K, Jaramillo S, Medina CA. Effects of PPAR-delta agonist and zinc on vaginal smooth muscle cells collagen and tropoelastin production. Int Urogynecol J 2012; 23: 1775-9.

11. Taneja SK, Kaur R. Pathology of ovary, uterus, vagina and gonadotrophs of female mice fed on Zn-deficient diet. Indian J Exp Biol 1990; 28: 105865.

12. Erekson EA, Yip SO, Wedderburn TS, Martin DK, Li FY, Choi JN, et al. The Vulvovaginal Symptoms Questionnaire: a questionnaire for measuring vulvovaginal symptoms in postmenopausal women. Menopause 2013; 20: 973-9.

13. Bachmann G, Cheng RJ, Rovner E. Vulvovaginal complaints. In: Lobo RA, editor. Treatment of the postmenopausal woman. Raven, New York: 1994.

14. Kim SH, Park ES, Kim TH. Rejuvenation using platelet-rich plasma and lipofilling for vaginal atrophy and lichen sclerosus. J Menopausal Med 2017; 23: 63-8.

15. Lee YK, Chung HH, Kim JW, Park NH, Song YS, Kang SB. Vaginal pH-balanced gel for the control of atrophic vaginitis among breast cancer survivors: a randomized controlled trial. Obstet Gynecol 2011; 117: 922-7.

16. Bygdeman M, Swahn ML. Replens versus dienoestrol cream in the symptomatic treatment of vaginal atrophy in postmenopausal women. Maturitas 1996; 23: 259-63.

17. Nachtigall LE. Comparative study: replens versus local estrogen in menopausal women. Fertil Steril 1994; 61: 178-80.

18. Dezzutti CS, Brown ER, Moncla B, Russo J, Cost M, Wang $\mathrm{L}$, et al. Is wetter better? An evaluation of over-the-counter personal lubricants for safety and anti-HIV-1 activity. PLoS One 2012; 7: e48328.

19. Houston DMJ, Robins B, Bugert JJ, Denyer SP, Heard CM. In vitro permeation and biological activity of punicalagin and zinc (II) across skin and mucous membranes prone to Herpes simplex virus infection. Eur J Pharm Sci 2017; 96: 99-106. 